\title{
On the different convex hulls of sets involving singular values
}

\author{
B. Dacorogna
}

Département de Mathématiques, Ecole Polytechnique Fédérale de Lausanne, CH 1015 Lausanne, Suisse e-mail: Bernard.Dacorogna@epfl.ch

\section{Tanteri}

Département de Mathématiques, Ecole Polytechnique Fédérale de Lausanne, CH 1015 Lausanne, Suisse e-mail: Chiara.Tanteri@epfl.ch

(MS received 2 June 1997. Revised MS received 5 December 1997)

We give a representation formula for the convex, polyconvex and rank one convex hulls of a set of $n \times n$ matrices with prescribed singular values.

\section{Introduction}

Let $\xi \in \mathbb{R}^{n \times n}$ and denote by $0 \leqq \lambda_{1}(\xi) \leqq \lambda_{2}(\xi) \leqq \ldots \leqq \lambda_{n}(\xi)$ the singular values of the matrix $\xi$ (i.e. the eigenvalues of $\left(\xi^{t} \xi\right)^{\frac{1}{2}}$; this implies in particular that $|\xi|^{2}=\sum_{i=1}^{n}\left[\lambda_{i}(\xi)\right]^{2}$ and $|\operatorname{det} \xi|=\prod_{i=1}^{n}\left[\lambda_{i}(\xi)\right]$. Let $0<a_{1} \leqq a_{2} \leqq \ldots \leqq a_{n}$ and

$$
E=\left\{\xi \in \mathbf{R}^{n \times n}: \lambda_{i}(\xi)=a_{i}, i=1, \ldots, n\right\} .
$$

The main results of this article (cf. Theorem 3.1) are that

$$
\begin{gathered}
c o E=\left\{\xi \in \mathbf{R}^{n \times n}: \sum_{i=v}^{n} \lambda_{i}(\xi) \leqq \sum_{i=v}^{n} a_{i}, v=1, \ldots, n\right\}, \\
P c o E=R \operatorname{RoE}=\left\{\xi \in \mathbf{R}^{n \times n}: \prod_{i=v}^{n} \lambda_{i}(\xi) \leqq \prod_{i=v}^{n} a_{i}, v=1, \ldots, n\right\},
\end{gathered}
$$

where $c o E$ denotes the convex hull of $E$, and $P c o E$ (respectively $R c o E$ ) the polyconvex (respectively the rank one convex) hull of $E$. The first notion corresponds to the classical one (cf. [9]) while the two others will be defined in Section 2.

It is interesting to note that, if $a_{1}=a_{2}=\ldots=a_{n}$, then it turns out that

$$
c o E=P \operatorname{coE}=R \operatorname{RoE}=\left\{\xi \in \mathbf{R}^{n \times n}: \lambda_{n}(\xi) \leqq a_{n}\right\}
$$

as already observed in $[4,6]$. The case where the $a_{i}$ are not all equal is more involved and has already been considered in $[5,7]$ when $n=2$.

An important application of the above representations is for attainment results in problems of the calculus of variations. A direct consequence of the results of [7] (in particular Theorems 6.1 and 6.4) leads to the following existence theorem: let $\Omega \subset \mathbf{R}^{n}$ 
be an open set, $a_{i}: \overline{\mathbf{\Omega}} \times \mathbf{R}^{n} \rightarrow \mathbf{R}, i=1, \ldots, n$ be continuous functions satisfying

$$
0<c \leqq a_{1}(x, s) \leqq \ldots \leqq a_{n}(x, s)
$$

for every $(x, s) \in \bar{\Omega} \times \mathbf{R}^{n}$ and let $\varphi \in C^{1}\left(\bar{\Omega} ; \mathbf{R}^{n}\right)$ satisfy

$$
\prod_{i=v}^{n} \lambda_{i}(D \varphi(x))<\prod_{i=v}^{n} a_{i}(x, \varphi(x)), \quad x \in \Omega, \quad v=1, \ldots, n,
$$

(in particular $\varphi \equiv 0)$; then there exists $u \in \mathbf{W}^{1, \infty}\left(\Omega ; \mathbf{R}^{n}\right)$ such that

$$
\left\{\begin{array}{l}
\lambda_{i}(D u(x))=a_{i}(x, u(x)), \quad \text { a.e. } x \in \Omega, \quad i=1, \ldots, n \\
u(x)=\varphi(x), \quad x \in \partial \Omega
\end{array}\right.
$$

\section{The different convex hulls}

Before proceeding with the proofs of our main results, we introduce the following definition and properties (cf. [7] for more details).

Definition 2.1. Let $E \subset \mathbf{R}^{m \times n}$ and

$$
F_{E}=\left\{f: \mathbf{R}^{m \times n} \rightarrow \overline{\mathbf{R}}=\mathbf{R} \cup\{+\infty\},\left.f\right|_{E}=0\right\} .
$$

Define

$$
c o E=\left\{\xi \in \mathbf{R}^{m \times n}: f(\xi) \leqq 0, \forall f \in F_{E}, f \text { convex }\right\},
$$

called the convex hull of $E$;

$$
P c o E=\left\{\xi \in \mathbf{R}^{m \times n}: f(\xi) \leqq 0, \forall f \in F_{E}, f \text { polyconvex }\right\},
$$

called the polyconvex hull of $E$;

$$
R \operatorname{coE}=\left\{\xi \in \mathbf{R}^{m \times n}: f(\xi) \leqq 0, \forall f \in F_{E}, f \text { rank one convex }\right\},
$$

called the rank one convex hull of $E$.

REMARK 2.2. The first one corresponds to the classical definition of convex hull (cf. [9]).

From the above definition, we can easily deduce the following propositions:

Proposition 2.3. Let $E \subset \mathbf{R}^{m \times n}$; then

$$
E \subset R \operatorname{coE} \subset P \operatorname{PoE} \subset \operatorname{coE} \text {. }
$$

Proposition 2.4. Let $E \subset \mathbf{R}^{m \times n}$ and define by induction

$$
R_{0} c o E=E \text {, }
$$

$$
R_{i+1} c o E=\left\{\xi \in \mathbf{R}^{m \times n}: \xi=t A+(1-t) B, t \in(0,1), A, B \in R_{i} c o E, \operatorname{rank}\{A-B\}=1\right\} .
$$

Then $R \operatorname{coE}=\cup_{i \in \mathrm{N}} R_{i} c o E$.

REMARK 2.5. We can observe that the above proposition is a weaker version of the result obtained in the characterisation of convex and polyconvex hulls. For example, using Carathéodory's Theorem, we have (cf. [9]):

$$
\operatorname{coE}=\left\{\xi \in \mathbf{R}^{m \times n}: \xi=\sum_{i=1}^{m n+1} t_{i} \xi_{i}, \xi_{i} \in E, t_{i} \geqq 0, \text { with } \sum_{i=1}^{m n+1} t_{i}=1\right\} .
$$


PROPOSITION 2.6. Let $0 \leqq \lambda_{1}(\xi) \leqq \lambda_{2}(\xi) \leqq \ldots \leqq \lambda_{n}(\xi)$ be the singular values of the matrix $\xi \in \mathbf{R}^{n \times n}$. Then

(i) $\xi \rightarrow \sum_{i=v}^{n} \lambda_{i}(\xi)$ is a convex function, for every $v=1, \ldots, n$;

(ii) $\xi \rightarrow \prod_{i=v}^{n} \lambda_{i}(\xi)$ is a polyconvex function, for every $v=1, \ldots, n$.

For a proof of the first result, we refer to $[2,3,8]$; for the last one, see [2] and [1], when $n=2$ and $n=3$ (the general case follows similarly).

\section{The main results}

In this section we will proceed with the proof of the main result of this article:

THEOREM 3.1. Let $\xi \in \mathbf{R}^{n \times n}$ and denote by $0 \leqq \lambda_{1}(\xi) \leqq \lambda_{2}(\xi) \leqq \ldots \leqq \lambda_{n}(\xi)$ the singular values of the matrix $\xi$. Let $0<a_{1} \leqq a_{2} \leqq \ldots \leqq a_{n}$,

$$
E=\left\{\xi \in \mathbf{R}^{n \times n}: \lambda_{i}(\xi)=a_{i}, i=1, \ldots, n\right\} .
$$

Then:

(i) $c o E=\left\{\xi \in \mathbf{R}^{n \times n}: \sum_{i=v}^{n} \lambda_{i}(\xi) \leqq \sum_{i=\nu}^{n} a_{i}, v=1, \ldots, n\right\}$;

(ii) $P \operatorname{coE}=R \operatorname{coE}=\left\{\xi \in \mathbf{R}^{n \times n}: \prod_{i=v}^{n} \lambda_{i}(\xi) \leqq \prod_{i=v}^{n} a_{i}, v=1, \ldots, n\right\}$;

(iii) intRcoE $=\left\{\xi \in \mathbf{R}^{n \times n}: \prod_{i=v}^{n} \lambda_{i}(\xi)<\prod_{i=v}^{n} a_{i}, v=1, \ldots, n\right\}$.

REMARK 3.2. When $n=2$ and $E=\left\{\xi \in \mathbf{R}^{2 \times 2}: \lambda_{1}(\xi)=a_{1}, \lambda_{2}(\xi)=a_{2}\right\}$, the theorem reads as

$$
c o E=\left\{\xi \in \mathbf{R}^{2 \times 2}: \lambda_{2}(\xi) \leqq a_{2}, \lambda_{1}(\xi)+\lambda_{2}(\xi) \leqq a_{1}+a_{2}\right\}
$$

and

$$
P \operatorname{coE}=R \operatorname{RoE}=\left\{\xi \in \mathbf{R}^{2 \times 2}: \lambda_{2}(\xi) \leqq a_{2}, \lambda_{1}(\xi) \cdot \lambda_{2}(\xi) \leqq a_{1} \cdot a_{2}\right\} .
$$

Proof of Theorem 3.1(i). Let $K=\left\{\xi \in \mathbf{R}^{n \times n}: \sum_{i=v}^{n} \lambda_{i}(\xi) \leqq \sum_{i=v}^{n} a_{i}, v=1, \ldots, n\right\}$.

We show that $c o E=K$. We divide the proof into two steps.

Step 1. $c o E \subset K$. The inclusion $c o E \subset K$ is easy. In fact, $E \subset K$ and from Proposition 2.6, the functions $\xi \rightarrow \sum_{i=v}^{n} \lambda_{i}(\xi)$ are convex. Therefore $K$ is convex and hence $c o E \subset K$.

Step 2. $K \subset \operatorname{coE}$. Let $\xi \in K$; we will prove that $\xi$ can be expressed as a convex combination of elements of $E$, i.e. $\zeta \in c o E$.

Since the functions $\xi \rightarrow \lambda_{i}(\xi)$ are invariant by orthogonal transformations, we can assume, without loss of generality, that

$$
\xi=\left(\begin{array}{lll}
x_{1} & & \\
& \ddots & \\
& & x_{n}
\end{array}\right),
$$

with $0 \leqq x_{1} \leqq x_{2} \leqq \ldots \leqq x_{n}$ and $\sum_{i=v}^{n} x_{i} \leqq \sum_{i=v}^{n} a_{i}, v=1, \ldots, n$.

We proceed by induction. We start with the proof in dimension $n=2$.

(i) $n=2$. We subdivide this case into two parts:

(a) $x_{1} \leqq a_{1}$ and, since $\xi \in K$, then $x_{2} \leqq a_{2}$ and $x_{1}+x_{2} \leqq a_{1}+a_{2}$.

Since $-a_{1} \leqq x_{1} \leqq a_{1}$, then $x_{1}=t a_{1}+(1-t)\left(-a_{1}\right)$ with $t=\left(x_{1}+a_{1}\right) / 2 a_{1}$. We can write:

$$
\xi=\left(\begin{array}{cc}
x_{1} & 0 \\
0 & x_{2}
\end{array}\right)=t\left(\begin{array}{cc}
a_{1} & 0 \\
0 & x_{2}
\end{array}\right)+(1-t)\left(\begin{array}{cc}
-a_{1} & 0 \\
0 & x_{2}
\end{array}\right) \text {. }
$$


We proceed similarly for $x_{2}$, i.e. $x_{2}=s a_{2}+(1-s)\left(-a_{2}\right)$, where $s=\left(x_{2}+a_{2}\right) / 2 a_{2}$. Thus we obtain

$$
\left(\begin{array}{cc} 
\pm a_{1} & 0 \\
0 & x_{2}
\end{array}\right)=s\left(\begin{array}{cc} 
\pm a_{1} & 0 \\
0 & +a_{2}
\end{array}\right)+(1-s)\left(\begin{array}{cc} 
\pm a_{1} & 0 \\
0 & -a_{2}
\end{array}\right) .
$$

Combining (3.1) and (3.2), we get that

$$
\xi=\left(\begin{array}{cc}
x_{1} & 0 \\
0 & x_{2}
\end{array}\right)=\sum_{i=1}^{I} t_{i} \xi_{i}
$$

with $\lambda_{1}\left(\xi_{i}\right)=a_{1}, \lambda_{2}\left(\xi_{i}\right)=a_{2}$ (i.e. $\left.\xi_{i} \in E\right)$. Therefore

$$
\xi \in c o E \text {. }
$$
that

(b) $x_{1} \geqq a_{1}$, i.e. since $\xi \in K, a_{1} \leqq x_{1} \leqq x_{2} \leqq a_{2}$ and $x_{1}+x_{2} \leqq a_{1}+a_{2}$. This implies

$$
a_{1} \leqq x_{1} \leqq a_{1}+a_{2}-x_{2} .
$$

In this case we just interpolate $x_{1}$ between $a_{1}$ and $a_{1}+a_{2}-x_{2}$, i.e.

$$
x_{1}=t a_{1}+(1-t)\left(a_{1}+a_{2}-x_{2}\right),
$$

which implies that

$$
\xi=\left(\begin{array}{cc}
x_{1} & 0 \\
0 & x_{2}
\end{array}\right)=t\left(\begin{array}{cc}
a_{1} & 0 \\
0 & x_{2}
\end{array}\right)+(1-t)\left(\begin{array}{cc}
a_{1}+a_{2}-x_{2} & 0 \\
0 & x_{2}
\end{array}\right) .
$$

The first matrix is treated in case (a). For the second matrix, we interpolate $x_{2}$ between $a_{1}$ and $a_{2}$, i.e. $x_{2}=s a_{2}+(1-s) a_{1}$, to obtain

$$
\left(\begin{array}{cc}
a_{1}+a_{2}-x_{2} & 0 \\
0 & x_{2}
\end{array}\right)=s\left(\begin{array}{cc}
a_{1} & 0 \\
0 & a_{2}
\end{array}\right)+(1-s)\left(\begin{array}{cc}
a_{2} & 0 \\
0 & a_{1}
\end{array}\right) .
$$

Combining (3.3) and (3.4), we have proved that

$$
\xi=\sum_{i=1}^{I} t_{i} \xi_{i},
$$

with $\lambda_{1}\left(\xi_{i}\right)=a_{1}, \lambda_{2}\left(\xi_{i}\right)=a_{2}$ (i.e. $\xi_{i} \in E$ ). Therefore $\xi \in c o E$. In conclusion, we have obtained, for $n=2$, that

$$
K \subset c o E .
$$

(ii) $n>2$. We suppose that the result has been established up to $n-1$, i.e. every $\xi$ such that $\sum_{i=v}^{n-1} \lambda_{i}(\xi) \leqq \sum_{i=v}^{n-1} a_{i}, v=1,2, \ldots, n-1$ (i.e. $\xi \in K$ ) can be expressed as a convex combination of elements of $\left\{\xi \in \mathbf{R}^{(n-1) \times(n-1)}: \lambda_{i}(\xi)=a_{i}, i=1, \ldots, n-1\right\}$, i.e.

$$
\xi=\sum_{\mu=1}^{I} t_{\mu} \xi_{\mu}
$$

with $\xi_{\mu}$ such that $\lambda_{i}\left(\xi_{\mu}\right)=a_{i}, i=1,2, \ldots,(n-1)$. We divide the proof into five parts:

Part $1.0 \leqq x_{1} \leqq x_{2} \leqq x_{1}+x_{2} \leqq a_{2}$. Note that these conditions imply that $x_{1}+x_{2} \leqq a_{1}+a_{2}$ and $x_{2} \leqq a_{2}$. We can therefore apply the case $n=2$ to $\left\{x_{1}, x_{2}\right\}$ and to $\left\{a_{1}, a_{2}\right\}$. We then use the hypothesis of induction on $\left\{x_{3}, \ldots, x_{n}\right\}$ and on $\left\{a_{3}, \ldots, a_{n}\right\}$. Combining these two decompositions, we get the result, i.e. $\xi \in c o E$. 
Part 2. $0 \leqq x_{1} \leqq x_{2} \leqq a_{2} \leqq x_{1}+x_{2}$. We can write

$$
\begin{aligned}
& \xi=\left(\begin{array}{llll}
x_{1} & & & \\
& x_{2} & & \\
& & \ddots & \\
& & & x_{n}
\end{array}\right)=\frac{1}{2}\left(\begin{array}{cccc}
x_{1} & \lambda & \\
\lambda & x_{2} & & \\
& & \ddots & \\
& & & x_{n}
\end{array}\right)+\frac{1}{2}\left(\begin{array}{cccc}
x_{1} & -\lambda & & \\
-\lambda & x_{2} & & \\
& & \ddots & \\
& & & x_{n}
\end{array}\right) \\
& =\frac{1}{2} A_{+}+\frac{1}{2} A_{-},
\end{aligned}
$$

where we have chosen

$$
\lambda^{2}=\left(x_{2}-a_{2}\right)\left(x_{1}-a_{2}\right) .
$$

Note that by hypothesis $\left(x_{1} \leqq x_{2} \leqq a_{2}\right)$ the right-hand side is positive. The choice of $\lambda$ allows us to find $O_{ \pm}, O_{ \pm}^{\prime} \in O(n)$ such that

$$
O_{ \pm} A_{ \pm} O_{ \pm}^{\prime}=\left(\begin{array}{ccccc}
a_{2} & & & & \\
& x_{1}+x_{2}-a_{2} & & & \\
& & x_{3} & & \\
& & & \ddots & \\
& & & & x_{n}
\end{array}\right) \text {. }
$$

We next apply the hypothesis of induction to

$$
\left\{y_{1}=x_{1}+x_{2}-a_{2}, y_{2}=x_{3}, \ldots, y_{n-1}=x_{n}\right\}
$$

and to

$$
\left\{b_{1}=a_{1}, b_{2}=a_{3}, \ldots, b_{n-1}=a_{n}\right\} .
$$

To do this, we first observe that

$$
0 \leqq y_{1}=x_{1}+x_{2}-a_{2} \leqq x_{1} \leqq x_{3}=y_{2} \leqq y_{3} \leqq \ldots \leqq y_{n-1}
$$

and

(1) if $v \geqq 2$, then $\sum_{i=v}^{n-1} y_{i}=\sum_{i=v+1}^{n} x_{i} \leqq \sum_{i=v+1}^{n} a_{i}=\sum_{i=v}^{n-1} b_{i}$;

(2) if $v=1$, then $\sum_{i=1}^{n-1} y_{i}=-a_{2}+\sum_{i=1}^{n} x_{i} \leqq-a_{2}+\sum_{i=1}^{n} a_{i}=\sum_{i=1}^{n-1} b_{i}$.

We can therefore deduce (by hypothesis of induction) that

$$
\left(\begin{array}{ccccc}
a_{2} & & & & \\
& x_{1}+x_{2}-a_{2} & & & \\
& & x_{3} & & \\
& & & \ddots & \\
& & & & x_{n}
\end{array}\right) \in c o E
$$

Since $c o E$ is invariant up to orthogonal transformations, we obtain that

$$
A_{ \pm}=\left(\begin{array}{cccc}
x_{1} & \pm \lambda & \\
\pm \lambda & x_{2} & & \\
& & \ddots & \\
& & & x_{n}
\end{array}\right) \in \operatorname{coE}
$$


which leads, combining (3.5) and (3.6), to

$$
\xi \in \operatorname{co} E,
$$

which is the claimed result.

Part 3. $x_{n-1} \geqq a_{n-1}$. We write

$$
\begin{aligned}
\xi=\left(\begin{array}{cccc}
x_{1} & & & \\
& \ddots & & \\
& & x_{n-1} & \\
& & & x_{n}
\end{array}\right) & =\frac{1}{2}\left(\begin{array}{cccc}
x_{1} & & & \\
& \ddots & & \\
& & x_{n-1} & \lambda \\
& \lambda & x_{n}
\end{array}\right)+\frac{1}{2}\left(\begin{array}{cccc}
x_{1} & & \\
& \ddots & & \\
& & x_{n-1} & -\lambda \\
& & -\lambda & x_{n}
\end{array}\right) \\
& =\frac{1}{2} A_{+}+\frac{1}{2} A_{-}
\end{aligned}
$$

where we have chosen

$$
\lambda^{2}=\left(x_{n}-a_{n-1}\right)\left(x_{n-1}-a_{n-1}\right) .
$$

Note that by hypothesis $\left(x_{n} \geqq x_{n-1} \geqq a_{n-1}\right)$ the right-hand side is positive. As above, the choice of $\lambda$ leads to the existence of $O_{ \pm}, O_{ \pm}^{\prime} \in O(n)$ such that

$$
O_{ \pm} A_{ \pm} O_{ \pm}^{\prime}=\left(\begin{array}{ccccc}
x_{1} & & & & \\
& \ddots & & & \\
& & x_{n-2} & & \\
& & & x_{n}+x_{n-1}-a_{n-1} & \\
& & & & a_{n-1}
\end{array}\right) .
$$

We next apply the hypothesis of induction to

$$
\left\{y_{1}=x_{1}, \ldots, y_{n-2}=x_{n-2}, y_{n-1}=x_{n}+x_{n-1}-a_{n-1}\right\}
$$

and to

$$
\left\{b_{1}=a_{1}, \ldots, b_{n-2}=a_{n-2}, b_{n-1}=a_{n}\right\} .
$$

To do this, we can observe that

$$
0 \leqq y_{1} \leqq \ldots \leqq y_{n-2}=x_{n-2} \leqq x_{n} \leqq x_{n}+x_{n-1}-a_{n-1}=y_{n-1} .
$$

By hypothesis and since $\xi \in K$, we have:

(1) if $v=n-1, y_{n-1}=x_{n}+x_{n-1}-a_{n-1} \leqq a_{n}$;

(2) if $1 \leqq v \leqq n-2$,

$$
\begin{aligned}
\sum_{i=v}^{n-1} y_{i} & =x_{n}+x_{n-1}-a_{n-1}+\sum_{i=v}^{n-2} x_{i}=-a_{n-1}+\sum_{i=v}^{n} x_{i} \\
& \leqq-a_{n-1}+\sum_{i=v}^{n} a_{i}=a_{n}+\sum_{i=v}^{n-2} a_{i}=\sum_{i=v}^{n-1} b_{i} .
\end{aligned}
$$

We can therefore deduce by hypothesis of induction and by invariance of $c o E$ under orthogonal transformations that

$$
A_{ \pm} \in c o E
$$


which combined with (3.7) lead to

$$
\xi \in \mathcal{C} O E \text {. }
$$

Part 4. $a_{2} \leqq x_{2} \leqq \ldots \leqq x_{n-1} \leqq a_{n-1}$. Note that this case occurs only if $n \geqq 4$. We first observe that we can therefore find $k \in\{2, \ldots, n-2\}$ such that

$$
a_{k} \leqq x_{k} \leqq x_{k+1} \leqq a_{k+1} \text {. }
$$

Hence we can write

$$
\xi=\left(\begin{array}{llll}
x_{1} & & & \\
& x_{2} & & \\
& & \ddots & \\
& & & x_{n}
\end{array}\right)=\frac{1}{2} A_{+}+\frac{1}{2} A_{-}
$$

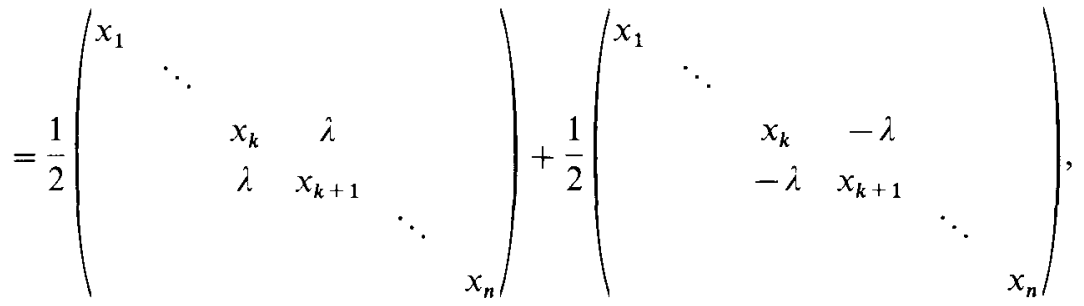

where we have chosen

$$
\hat{\lambda}^{2}=\left(x_{k}-b\right)\left(x_{k+1}-b\right)
$$

with $b=a_{k}$ (Part 4.1) or $b=a_{k+1}$ (Part 4.2). Note that, from the above assumption (3.8), the right-hand side is positive in both cases.

Part 4.1. $\left\{\begin{array}{l}a_{k} \leqq x_{k} \leqq x_{k+1} \leqq a_{k+1} \\ x_{k}+x_{k+1}+\sum_{i=v+1}^{n} x_{i} \leqq a_{k}+\sum_{i=v}^{n} a_{i}, \quad v=k+2, \ldots, n\end{array}\right.$

(with the convention $\sum_{i=n+1}^{n} x_{i}=0$ ).

$$
\text { Part 4.2. }\left\{\begin{array}{l}
a_{k} \leqq x_{k} \leqq x_{k+1} \leqq a_{k+1} \\
\sum_{i=\mu}^{k-1} x_{i}+\sum_{i=k+2}^{n} x_{i} \leqq \sum_{i=\mu+1}^{k} a_{i}+\sum_{i=k+2}^{n} a_{i}, \mu=1, \ldots, k-1 .
\end{array}\right.
$$

Before proceeding with the study of the above cases, we show that Part 4.1 and Part 4.2 cover all possibilities. In fact, if $0 \leqq x_{1} \leqq \ldots \leqq x_{n}$ and if $\sum_{i=v}^{n} x_{i} \leqq \sum_{i=v}^{n} a_{i}$, $v=1, \ldots, n$, then at least one of the following sets of inequalities holds:

$$
\begin{gathered}
x_{k}+x_{k+1}+\sum_{i=v+1}^{n} x_{i} \leqq a_{k}+\sum_{i=v}^{n} a_{i}, \quad v=k+2, \ldots, n ; \\
\sum_{i=\mu}^{k-1} x_{i}+\sum_{i=k+2}^{n} x_{i} \leqq \sum_{i=\mu+1}^{k} a_{i}+\sum_{i=k+2}^{n} a_{i}, \quad \mu=1, \ldots, k-1 .
\end{gathered}
$$

We proceed by contradiction and we assume that there exists $v \in\{k+2, \ldots, n\}$ and 
$\mu \in\{1, \ldots, k-1\}$ such that

$$
\begin{gathered}
x_{k}+x_{k+1}+\sum_{i=v+1}^{n} x_{i}>a_{k}+\sum_{i=v}^{n} a_{i}, \\
\sum_{i=\mu}^{k-1} x_{i}+\sum_{i=k+2}^{n} x_{i}>\sum_{i=\mu+1}^{k} a_{i}+\sum_{i=k+2}^{n} a_{i} .
\end{gathered}
$$

Summing up these two inequalities and using the assumptions, we get

$$
\sum_{i=\mu}^{n} a_{i}+\sum_{i=v+1}^{n} a_{i} \geqq \sum_{i=\mu}^{n} x_{i}+\sum_{i=v+1}^{n} x_{i}>a_{k}-a_{k+1}+\sum_{i=\mu+1}^{n} a_{i}+\sum_{i=v}^{n} a_{i}
$$

i.e.

$$
a_{\mu}+a_{k+1}>a_{k}+a_{v} .
$$

However, $\mu \in\{1, \ldots, k-1\}$, hence $a_{\mu} \leqq a_{k}$ and $v \in\{k+2, \ldots, n\}$, therefore $a_{v} \geqq a_{k+1}$. We therefore get

$$
a_{k}+a_{k+1} \geqq a_{\mu}+a_{k+1}>a_{k}+a_{v} \geqq a_{k}+a_{k+1},
$$

which is the claimed contradiction. In conclusion, Part 4.1 and Part 4.2 cover all possibilities. We now separately study these two cases:

$$
\text { Part 4.1. }\left\{\begin{array}{l}
a_{k} \leqq x_{k} \leqq x_{k+1} \leqq a_{k+1} \\
x_{k}+x_{k+1}+\sum_{i=v+1}^{n} x_{i} \leqq a_{k}+\sum_{i=v}^{n} a_{i}, \quad v=k+2, \ldots, n
\end{array}\right.
$$

(with the convention $\sum_{i=n+1}^{n} x_{i}=0$ ). We choose here $b=a_{k}$ in (3.9) and (3.10). We can, as above, find $O_{ \pm}, O_{ \pm}^{\prime} \in O(n)$ such that

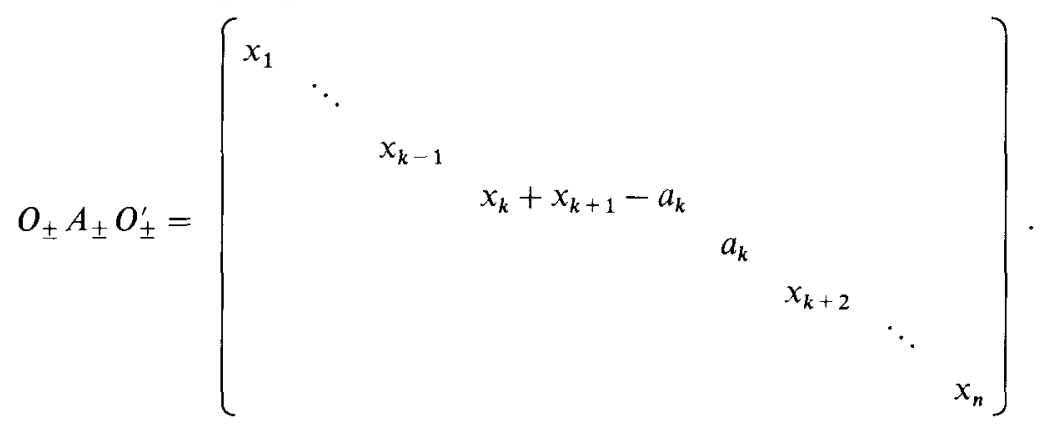

We apply the hypothesis of induction to

$$
\left\{y_{1}=x_{1}, \ldots, y_{k-1}=x_{k-1}, y_{k}=x_{k}+x_{k+1}-a_{k}, y_{k+1}=x_{k+2}, \ldots, y_{n-1}=x_{n}\right\}
$$

and to

$$
\left\{b_{1}=a_{1}, \ldots, b_{k-1}=a_{k-1}, b_{k}=a_{k+1}, \ldots, b_{n-1}=a_{n}\right\} .
$$

Observe that, since $a_{k} \leqq x_{k}$, then $0 \leqq y_{1} \leqq \ldots \leqq y_{k-1}=x_{k-1} \leqq x_{k}+x_{k+1}-a_{k}=y_{k}$. On the contrary, a priori, we cannot compare $y_{k}$ to $y_{k+1} \leqq \ldots \leqq y_{n-1}$. We next verify the hypothesis of induction.

(1) Let $v=n-1$. We must show that $y_{n-1}=x_{n} \leqq b_{n-1}=a_{n}$ and $y_{k} \leqq b_{n-1}=a_{n}$. The first inequality is valid by assumption, while the second is also true since it is equivalent to $x_{k}+x_{k+1} \leqq a_{k}+a_{n}$ which is the assumption of Part 4.1 with $v=n$. 
(2) Let $n-2 \geqq v \geqq k+1$. We have again by hypothesis of Part 4.1 and since $\xi \in K$

$$
\left\{\begin{array}{l}
\sum_{i=v}^{n-1} y_{i}=\sum_{i=v+1}^{n} x_{i} \leqq \sum_{i=v+1}^{n} a_{i}=\sum_{i=v}^{n-1} b_{i} \\
y_{k}+\sum_{i=v+1}^{n-1} y_{i}=x_{k}+x_{k+1}-a_{k}+\sum_{i=v+2}^{n} x_{i} \leqq \sum_{i=v+1}^{n} a_{i}=\sum_{i=v}^{n-1} b_{i} .
\end{array}\right.
$$

(3) If $k \geqq v \geqq 1$,

$$
\sum_{i=v}^{n-1} y_{i}=\sum_{i=v}^{k-1} y_{i}+\sum_{i=k}^{n-1} y_{i}=\sum_{i=v}^{k-1} x_{i}+\sum_{i=k}^{n} x_{i}-a_{k} \leqq \sum_{i=v}^{n} a_{i}-a_{k}=\sum_{i=v}^{n-1} b_{i} .
$$

Therefore we can apply the hypothesis of induction and the invariance of $c o E$ under orthogonal transformations to get

$$
A_{ \pm} \in \mathcal{c o E} \text {. }
$$

Combining (3.9) and (3.11), we indeed get that

$$
\xi \in c o E \text {. }
$$

$$
\text { Part 4.2. }\left\{\begin{array}{l}
a_{k} \leqq x_{k} \leqq x_{k+1} \leqq a_{k+1}, \\
\sum_{i=\mu}^{k-1} x_{i}+\sum_{i=k+2}^{n} x_{i} \leqq \sum_{i=\mu+1}^{k} a_{i}+\sum_{i=k+2}^{n} a_{i}, \quad \mu=1, \ldots, k-1 .
\end{array}\right.
$$

We choose here $b=a_{k+1}$ in (3.9) and (3.10). We can, as above, find $O_{ \pm}, O_{ \pm}^{\prime} \in O(n)$ such that

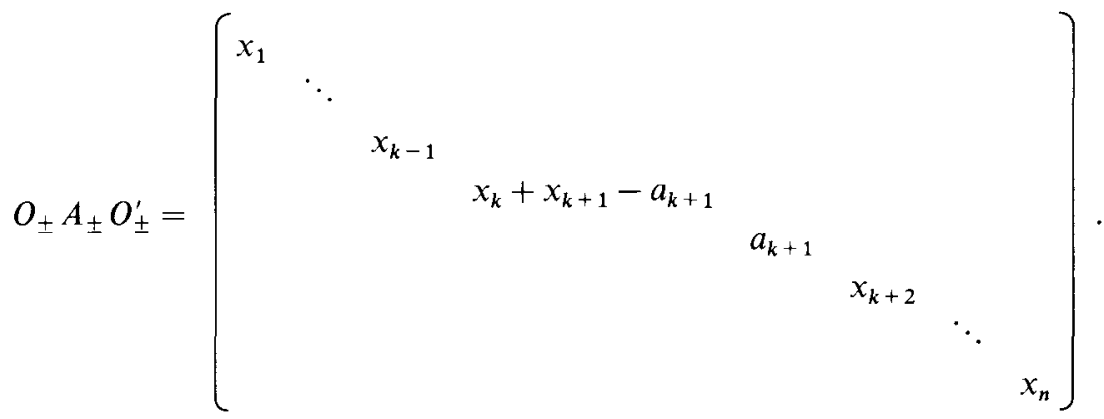

We apply the hypothesis of induction to

$$
\left\{y_{1}=x_{1}, \ldots, y_{k-1}=x_{k-1}, y_{k}=x_{k}+x_{k+1}-a_{k+1}, y_{k+1}=x_{k+2}, \ldots, y_{n-1}=x_{n}\right\}
$$

and to

$$
\left\{b_{1}=a_{1}, \ldots, b_{k-1}=a_{k-1}, b_{k}=a_{k}, b_{k+1}=a_{k+2}, \ldots, b_{n-1}=a_{n}\right\} .
$$

Observe that, since $x_{k+1} \leqq a_{k+1}$, we have $y_{k}=x_{k}+x_{k+1}-a_{k+1} \leqq x_{k} \leqq x_{k+2}=$ $y_{k+1} \leqq \ldots \leqq y_{n-1}$. On the contrary, a priori, we cannot compare $y_{k}$ to $0 \leqq y_{1} \leqq \ldots \leqq y_{k-1}$. We next verify the hypothesis of induction. Since $\xi \in K$ and by assumption of Part 4.2, we get:

(1) if $v \geqq k+1, \sum_{i=v}^{n-1} y_{i}=\sum_{i=v+1}^{n} x_{i} \leqq \sum_{i=v+1}^{n} a_{i}=\sum_{i=v}^{n-1} b_{i}$; 
(2) if $v=k$,

$$
\left\{\begin{array}{l}
\sum_{i=k}^{n-1} y_{i}=-a_{k+1}+\sum_{i=k}^{n} x_{i} \leqq-a_{k+1}+\sum_{i=k}^{n} a_{i}=\sum_{i=k}^{n-1} b_{i}, \\
y_{k-1}+\sum_{i=k+1}^{n-1} y_{i}=x_{k-1}+\sum_{i=k+2}^{n} x_{i} \leqq a_{k}+\sum_{i=k+2}^{n} a_{i}=\sum_{i=k}^{n-1} b_{i}
\end{array}\right.
$$

(3) if $k-1 \geqq v \geqq 1$,

$$
\left\{\begin{aligned}
\sum_{i=v}^{n-1} y_{i} & =-a_{k+1}+\sum_{i=v}^{n} x_{i} \leqq-a_{k+1}+\sum_{i=v}^{n} a_{i}=\sum_{i=v}^{n-1} b_{i}, \\
\sum_{i=v-1}^{k-1} y_{i} & +\sum_{i=k+1}^{n-1} y_{i}=\sum_{i=v-1}^{k-1} x_{i}+\sum_{i=k+2}^{n} x_{i} \leqq \sum_{i=v}^{k} a_{i}+\sum_{i=k+2}^{n} a_{i} \\
& =\sum_{i=v}^{n-1} b_{i} .
\end{aligned}\right.
$$

We can therefore apply the hypothesis of induction to obtain

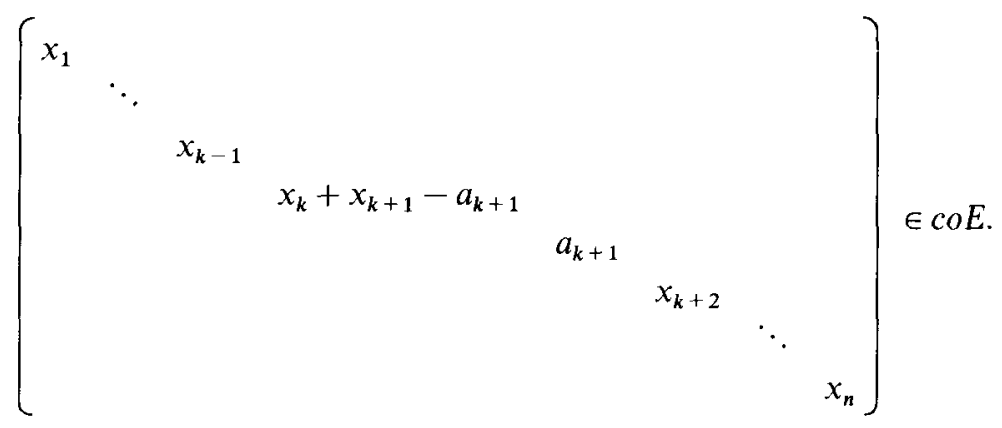

The invariance under orthogonal transformations leads immediately to

$$
A_{ \pm} \in \operatorname{coE} \text {. }
$$

Combining (3.9) and (3.12), we have indeed obtained

$$
\xi \in \operatorname{coE} \text {. }
$$

This achieves the proof of Step 2, i.e. $K \subset c o E$, and thus part (i) of the theorem.

Proof of Theorem 3.1(ii). Let $X=\left\{\xi \in \mathbf{R}^{n \times n}: \prod_{i=v}^{n} \lambda_{i}(\xi) \leqq \prod_{i=v}^{n} a_{i}, v=1, \ldots, n\right\}$. We prove that $X=R c o E$. We divide the proof into two steps.

Step 1. RcoE $\subset X$. Observe that $E \subset X$ and, from Proposition 2.6, the functions $\xi \rightarrow \Pi_{i=\nu}^{n} \lambda_{i}(\xi), v=1, \ldots, n$ are polyconvex (and hence rank one convex). Therefore we deduce that $X$ is polyconvex and hence

$$
R \operatorname{coE} \subset P \operatorname{PcoE} \subset X .
$$

Step 2. $X \subset R c o E$. Let $\xi \in X$; we will prove that $\xi \in R c o E$. Since the functions $\xi \rightarrow \lambda_{i}(\xi)$ are invariant by orthogonal transformations, we can assume, without loss 
of generality, that

$$
\xi=\left(\begin{array}{lll}
x_{1} & & \\
& \ddots & \\
& & x_{n}
\end{array}\right)
$$

with $0 \leqq x_{1} \leqq x_{2} \leqq \ldots \leqq x_{n}$ and $\Pi_{i=v}^{n} x_{i} \leqq \Pi_{i=v}^{n} a_{i}, v=1, \ldots, n$.

We show the result by induction. We start with the proof in dimension $n=2$. Note that the proof of this case is simpler than the one in [6].

(i) $n=2$. We write

$$
\xi=\left(\begin{array}{cc}
x_{1} & 0 \\
0 & x_{2}
\end{array}\right)=\frac{1}{2}\left(\begin{array}{cc}
x_{1} & \lambda \\
0 & x_{2}
\end{array}\right)+\frac{1}{2}\left(\begin{array}{cc}
x_{1} & -\lambda \\
0 & x_{2}
\end{array}\right)=\frac{1}{2} A_{+}+\frac{1}{2} A_{-}
$$

(observe that $\left.\operatorname{rank}\left\{A_{+}-A_{-}\right\} \leqq 1\right\}$ and we choose

$$
\lambda^{2}=\frac{\left(a_{2}^{2}-x_{2}^{2}\right)\left(a_{2}^{2}-x_{1}^{2}\right)}{a_{2}^{2}} .
$$

Note that the right-hand side is positive by assumption $\left(0 \leqq x_{1} \leqq x_{2} \leqq a_{2}\right)$. This leads to

$$
\lambda_{1}\left(A_{ \pm}\right)=\frac{x_{1} x_{2}}{a_{2}}, \quad \lambda_{2}\left(A_{ \pm}\right)=a_{2} .
$$

Therefore $\exists O_{ \pm}, O_{ \pm}^{\prime} \in O(2)$ such that

$$
O_{ \pm} A_{ \pm} O_{ \pm}^{\prime}=\left(\begin{array}{cc}
\frac{x_{1} x_{2}}{a_{2}} & 0 \\
0 & a_{2}
\end{array}\right)
$$

However, we have

$$
\left(\begin{array}{cc}
\frac{x_{1} x_{2}}{a_{2}} & 0 \\
0 & a_{2}
\end{array}\right)=\left(\frac{1}{2}+\frac{x_{1} x_{2}}{2 a_{1} a_{2}}\right)\left(\begin{array}{cc}
a_{1} & 0 \\
0 & a_{2}
\end{array}\right)+\left(\frac{1}{2}-\frac{x_{1} x_{2}}{2 a_{1} a_{2}}\right)\left(\begin{array}{cc}
-a_{1} & 0 \\
0 & a_{2}
\end{array}\right)
$$

and hence

$$
\left(\begin{array}{cc}
\frac{x_{1} x_{2}}{a_{2}} & 0 \\
0 & a_{2}
\end{array}\right) \in R_{1} \operatorname{coE} \subset \operatorname{Rco} E
$$

Since $R c o E$ is invariant up to orthogonal transformations, we deduce that

$$
A_{ \pm}=\left(\begin{array}{cc}
x_{1} & \pm \lambda \\
0 & x_{2}
\end{array}\right) \in R \operatorname{Ro} E
$$


Finally, combining (3.13) and (3.14), we obtain that

$$
\xi=\left(\begin{array}{cc}
x_{1} & 0 \\
0 & x_{2}
\end{array}\right) \in R \operatorname{Ro} E
$$

which is the claimed result.

(ii) $n>2$. We divide this case into four parts.

Part 1. $x_{2} \leqq a_{2}$. We write

$$
\begin{aligned}
\xi=\left(\begin{array}{cccc}
x_{1} & & & \\
& x_{2} & & \\
& & \ddots & \\
& & & x_{n}
\end{array}\right) & =\frac{1}{2}\left(\begin{array}{cccc}
x_{1} & \lambda & & \\
0 & x_{2} & & \\
& & \ddots & \\
& & & x_{n}
\end{array}\right)+\frac{1}{2}\left(\begin{array}{cccc}
x_{1} & -\lambda & & \\
0 & x_{2} & & \\
& & \ddots & \\
& & & x_{n}
\end{array}\right) \\
& =\frac{1}{2} A_{+}+\frac{1}{2} A_{-}
\end{aligned}
$$

(observe that $\operatorname{rank}\left\{A_{+}-A_{-}\right\} \leqq 1$ ) and we define $\lambda$ by:

$$
\lambda^{2}=\frac{\left(a_{2}^{2}-x_{2}^{2}\right)\left(a_{2}^{2}-x_{1}^{2}\right)}{a_{2}^{2}} .
$$

Note that the right-hand side is positive by assumption $\left(0 \leqq x_{1} \leqq x_{2} \leqq a_{2}\right)$. The choice of $\lambda$ (as in the case $n=2$ ) leads to the existence of $O_{ \pm}, O_{ \pm}^{\prime} \in O(n)$ such that

$$
O_{ \pm} A_{ \pm} O_{ \pm}^{\prime}=\left(\begin{array}{ccccc}
a_{2} & & & & \\
& \frac{x_{1} x_{2}}{a_{2}} & & & \\
& & x_{3} & & \\
& & & \ddots & \\
& & & & x_{n}
\end{array}\right) \text {. }
$$

We apply the hypothesis of induction to

$$
\left\{y_{1}=\frac{x_{1} x_{2}}{a_{2}}, y_{2}=x_{3}, \ldots, y_{n-1}=x_{n}\right\}
$$

and to

$$
\left\{b_{1}=a_{1}, b_{2}=a_{3}, \ldots, b_{n-1}=a_{n}\right\} .
$$

Note that, since $x_{2} \leqq a_{2}$, then $0 \leqq y_{1} \leqq \ldots \leqq y_{n-1}$.

We have to show that $\prod_{i=v}^{n-1} y_{i} \leqq \prod_{i=v}^{n-1} b_{i}, v=1, \ldots, n-1$.

(1) By assumption, if $v \geqq 2$, we have $\prod_{i=v}^{n-1} y_{i}=\prod_{i=v+1}^{n} x_{i} \leqq \prod_{i=v+1}^{n} a_{i}=\prod_{i=v}^{n-1} b_{i}$.

(2) If $v=1$, we have

$$
\prod_{i=1}^{n-1} y_{i}=\frac{x_{1} x_{2}}{a_{2}} \prod_{i=3}^{n} x_{i}=\frac{1}{a_{2}} \prod_{i=1}^{n} x_{i} \leqq \frac{1}{a_{2}} \prod_{i=1}^{n} a_{i}=a_{1} \prod_{i=3}^{n} a_{i}=\prod_{i=1}^{n-1} b_{i} .
$$


Therefore we can deduce that (by hypothesis of induction)

$$
\left(\begin{array}{ccccc}
a_{2} & & & & \\
& \frac{x_{1} x_{2}}{a_{2}} & & & \\
& & x_{3} & & \\
& & & \ddots & \\
& & & & x_{n}
\end{array}\right) \in R \operatorname{RoE} .
$$

Since $R c o E$ is invariant up to orthogonal transformations, we obtain

$$
A_{ \pm}=\left(\begin{array}{cccc}
x_{1} & \pm \lambda & & \\
0 & x_{2} & & \\
& & \ddots & \\
& & & x_{n}
\end{array}\right) \in R \operatorname{coE}
$$

and therefore, combining (3.15) and (3.16), we get

$$
\xi \in R \operatorname{coE} \text {, }
$$

which is the claimed result.

Part 2. $x_{n-1} \geqq a_{n-1}$. We write (as in Part 1, but interchanging the role of $\left(x_{n}, x_{n-1}\right)$ and $\left.\left(x_{1}, x_{2}\right)\right)$

$$
\begin{aligned}
& \xi=\left(\begin{array}{cccc}
x_{1} & & & \\
& \ddots & & \\
& & x_{n-1} & \\
& & & x_{n}
\end{array}\right)=\frac{1}{2}\left(\begin{array}{cccc}
x_{1} & & & \\
& \ddots & & \\
& & x_{n-1} & \lambda \\
& & 0 & x_{n}
\end{array}\right)+\frac{1}{2}\left(\begin{array}{cccc}
x_{1} & & & \\
& \ddots & & \\
& & x_{n-1} & -\lambda \\
& 0 & x_{n}
\end{array}\right) \\
& =\frac{1}{2} A_{+}+\frac{1}{2} A_{-}
\end{aligned}
$$

(observe that $\operatorname{rank}\left\{A_{+}-A_{-}\right\} \leqq 1$ ) and we choose $\lambda$ to be:

$$
\lambda^{2}=\frac{\left(x_{n}^{2}-a_{n-1}^{2}\right)\left(x_{n-1}^{2}-a_{n-1}^{2}\right)}{a_{n-1}^{2}} .
$$

Note that the right-hand side is positive by assumption $\left(a_{n-1} \leqq x_{n-1} \leqq x_{n}\right)$. As above, the choice of $\lambda$ leads to the existence of $O_{ \pm}, O_{ \pm}^{\prime} \in O(n)$ such that

$$
O_{ \pm} A_{ \pm} O_{ \pm}^{\prime}=\left(\begin{array}{cccccc}
x_{1} & & & & & \\
& x_{2} & & & & \\
& & \ddots & & \\
& & & x_{n-2} & \\
& & & & \frac{x_{n-1} x_{n}}{a_{n-1}} & \\
& & & & & a_{n-1}
\end{array}\right) \text {. }
$$

We apply the hypothesis of induction to

$$
\left\{y_{1}=x_{1}, \ldots, y_{n-2}=x_{n-2}, y_{n-1}=\frac{x_{n-1} x_{n}}{a_{n-1}}\right\}
$$


and to

$$
\left\{b_{1}=a_{1}, \ldots, b_{n-2}=a_{n-2}, b_{n-1}=a_{n}\right\} .
$$

Note that, since $a_{n-1} \leqq x_{n-1}$, then $0 \leqq y_{1} \leqq \ldots \leqq y_{n-1}$.

We verify the hypothesis of induction.

(1) First, observe that $y_{n-1} \leqq b_{n-1}$ because $x_{n-1} x_{n} \leqq a_{n-1} a_{n}$.

(2) If $1 \leqq v \leqq n-1$, then

$$
\begin{aligned}
\prod_{i=v}^{n-1} y_{i} & =\prod_{i=v}^{n-2} y_{i} \cdot y_{n-1}=\prod_{i=v}^{n-2} x_{i} \frac{x_{n-1} x_{n}}{a_{n-1}}=\frac{1}{a_{n-1}} \prod_{i=v}^{n} x_{i} \\
& \leqq \frac{1}{a_{n-1}} \prod_{i=v}^{n} a_{i}=\prod_{i=v}^{n-2} a_{i} \cdot a_{n}=\prod_{i=v}^{n-1} b_{i} .
\end{aligned}
$$

Therefore (by hypothesis of induction)

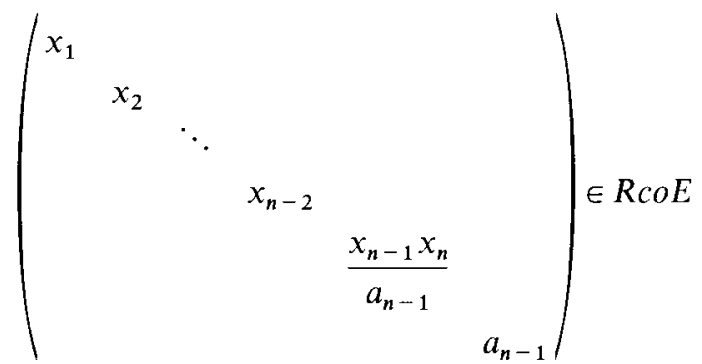

and, since $R \operatorname{coE}$ is invariant up orthogonal transformations, we obtain that $A_{ \pm} \in R c o E$, which combined with (3.17) leads to the claimed result,

$$
\xi \in R \operatorname{coE} \text {. }
$$

Part 3. $a_{2} \leqq x_{2} \leqq \ldots \leqq x_{n-1} \leqq a_{n-1}$. Note that this case occurs only if $n \geqq 4$. We first observe that we can therefore find $k \in\{2, \ldots, n-2\}$ such that

$$
a_{k} \leqq x_{k} \leqq x_{k+1} \leqq a_{k+1} .
$$

Hence we can write

$$
\xi=\left(\begin{array}{llll}
x_{1} & & & \\
& x_{2} & & \\
& & \ddots & \\
& & & x_{n}
\end{array}\right)=\frac{1}{2} A_{+}+\frac{1}{2} A_{-}
$$

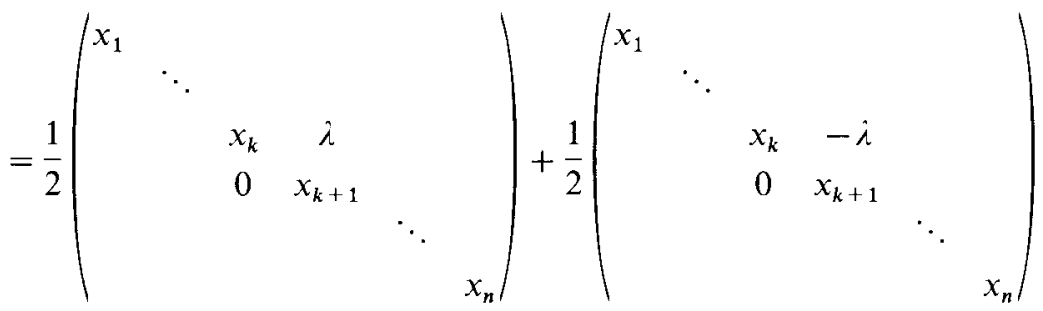


(observe that $\operatorname{rank}\left\{A_{+}-A_{-}\right\} \leqq 1$ ) where $\lambda$ is given by

$$
\lambda^{2}=\frac{\left(b^{2}-x_{k}^{2}\right)\left(b^{2}-x_{k+1}^{2}\right)}{b^{2}}
$$

where $b=a_{k}$ (Part 3.1) or $b=a_{k+1}$ (Part 3.2). Note that, from the above assumptions (3.18), the right-hand side is positive in both cases.

Part 3.1. $\left\{\begin{array}{l}a_{k} \leqq x_{k} \leqq x_{k+1} \leqq a_{k+1}, \\ x_{k} x_{k+1} \prod_{i=v+1}^{n} x_{i} \leqq a_{k} \prod_{i=v}^{n} a_{i}, \quad v=k+2, \ldots, n\end{array}\right.$

(with the convention $\prod_{i=n+1}^{n} x_{i}=1$ ).

Part 3.2. $\left\{\begin{array}{l}a_{k} \leqq x_{k} \leqq x_{k+1} \leqq a_{k+1}, \\ \prod_{i=\mu}^{k-1} x_{i} \cdot \prod_{i=k+2}^{n} x_{i} \leqq \prod_{i=\mu+1}^{k} a_{i} \cdot \prod_{i=k+2}^{n} a_{i}, \mu=1, \ldots, k-1 .\end{array}\right.$

Before proceeding with the study of the above cases, we show that Part 3.1 and Part 3.2 cover all possibilities. In fact, if $0 \leqq x_{1} \leqq \ldots \leqq x_{n}$ and if $\Pi_{i=v}^{n} x_{i} \leqq \Pi_{i=v}^{n} a_{i}$, $v=1, \ldots, n$, then at least one of the following sets of inequalities holds:

$$
\begin{gathered}
x_{k} x_{k+1} \prod_{i=v+1}^{n} x_{i} \leqq a_{k} \prod_{i=v}^{n} a_{i}, \quad v=k+2, \ldots, n \\
\prod_{i=\mu}^{k-1} x_{i} \cdot \prod_{i=k+2}^{n} x_{i} \leqq \prod_{i=\mu+1}^{k} a_{i} \cdot \prod_{i=k+2}^{n} a_{i}, \quad \mu=1, \ldots, k-1 .
\end{gathered}
$$

We proceed by contradiction and we assume that there exist $v \in\{k+2, \ldots, n\}$ and $\mu \in\{1, \ldots, k-1\}$ such that

$$
\begin{gathered}
x_{k} x_{k+1} \prod_{i=v+1}^{n} x_{i}>a_{k} \prod_{i=v}^{n} a_{i}, \\
\prod_{i=\mu}^{k-1} x_{i} \cdot \prod_{i=k+2}^{n} x_{i}>\prod_{i=\mu+1}^{k} a_{i} \cdot \prod_{i=k+2}^{n} a_{i} .
\end{gathered}
$$

Multiplying together the two inequalities and using the assumptions, we deduce that

$$
\prod_{i=\mu}^{n} a_{i} \cdot \prod_{i=\nu+1}^{n} a_{i} \geqq \prod_{i=\mu}^{n} x_{i} \cdot \prod_{i=v+1}^{n} x_{i}>a_{k} \prod_{i=v}^{n} a_{i} \cdot \prod_{i=\mu+1}^{k} a_{i} \cdot \prod_{i=k+2}^{n} a_{i},
$$

i.e.

$$
a_{\mu} \prod_{i=k+1}^{n} a_{i} \cdot \prod_{i=v+1}^{n} a_{i}>a_{k} \prod_{i=v}^{n} a_{i} \cdot \prod_{i=k+2}^{n} a_{i}
$$

therefore

$$
a_{\mu} a_{k+1}>a_{k} a_{v} .
$$

However, $\mu \in\{1, \ldots, k-1\}$, hence $a_{\mu} \leqq a_{k}$ and $v \in\{k+2, \ldots, n\}$, therefore $a_{v} \geqq a_{k+1}$. We therefore get

$$
a_{k} a_{k+1} \geqq a_{\mu} a_{k+1}>a_{k} a_{v} \geqq a_{k} a_{k+1},
$$

which is the claimed contradiction. In conclusion, Part 3.1 and Part 3.2 cover all 
possibilities. We now study these two cases separately.

$$
\text { Part } 3.1\left\{\begin{array}{l}
a_{k} \leqq x_{k} \leqq x_{k+1} \leqq a_{k+1}, \\
x_{k} x_{k+1} \prod_{i=v+1}^{n} x_{i} \leqq a_{k} \prod_{i=v}^{n} a_{i} \quad v=k+2, \ldots, n
\end{array}\right.
$$

(with the convention $\prod_{i=n+1}^{n} x_{i}=1$ ). We choose here $b=a_{k}$ in (3.19) and (3.20); therefore we can find $O_{ \pm}, O_{ \pm}^{\prime} \in O(n)$ such that

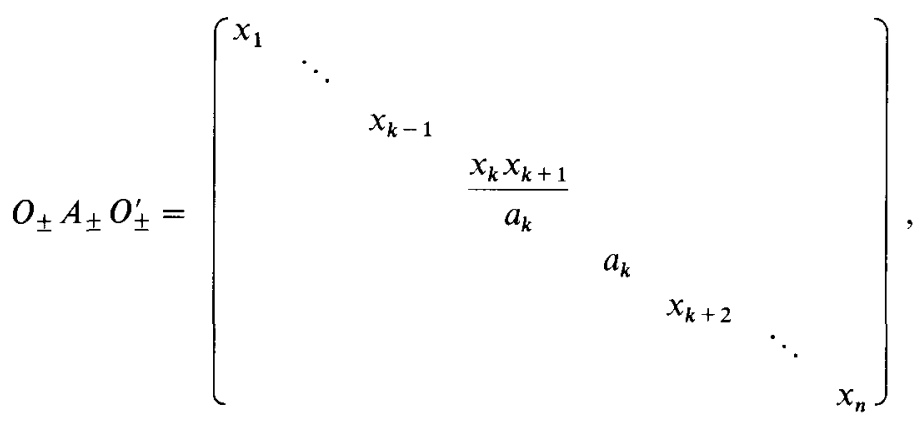

where we recall that

$$
A_{ \pm}=\left(\begin{array}{cccccc}
x_{1} & & & & & \\
& \ddots & & & & \\
& & x_{k} & \pm \lambda & & \\
& & 0 & x_{k+1} & & \\
& & & & \ddots & \\
& & & & & x_{n}
\end{array}\right) .
$$

We apply the hypothesis of induction to

$$
\left\{y_{1}=x_{1}, \ldots, y_{k-1}=x_{k-1}, y_{k}=\frac{x_{k} x_{k+1}}{a_{k}}, y_{k+1}=x_{k+2}, \ldots, y_{n-1}=x_{n}\right\}
$$

and to

$$
\left\{b_{1}=a_{1}, \ldots, b_{k-1}=a_{k-1}, b_{k}=a_{k+1}, \ldots, b_{n-1}=a_{n}\right\} .
$$

Observe that, since $a_{k} \leqq x_{k}$, then $0 \leqq y_{1} \leqq \ldots \leqq y_{k-1} \leqq y_{k}$. On the contrary, a priori, we cannot compare $y_{k}$ to $y_{k+1} \leqq \ldots \leqq y_{n-1}$. We next verify the hypothesis of induction.

(1) We must show that $x_{n}=y_{n-1} \leqq b_{n-1}=a_{n}$ and $y_{k} \leqq b_{n-1}=a_{n}$.

The first inequality is verified by assumption and the second is also verified by the assumption of Part 3.1 with $v=n$. The assumption $\xi \in X$ and that of Part 3.1 again ensure that

(2) if $n-1 \geqq v \geqq k+1$,

$$
\left\{\begin{array}{l}
\prod_{i=v}^{n-1} y_{i}=\prod_{i=v+1}^{n} x_{i} \leqq \prod_{i=v+1}^{n} a_{i}=\prod_{i=v}^{n-1} b_{i}, \\
y_{k} \prod_{i=v+1}^{n-1} y_{i}=\frac{x_{k} x_{k+1}}{a_{k}} \cdot \prod_{i=v+2}^{n} x_{i} \leqq \prod_{i=v+1}^{n} a_{i}=\prod_{i=v}^{n-1} b_{i} .
\end{array}\right.
$$


(3) If $k \geqq v \geqq 1$,

$$
\begin{aligned}
\prod_{i=v}^{n-1} y_{i} & =\prod_{i=v}^{k} y_{i} \cdot \prod_{i=k+1}^{n-1} y_{i}=\frac{1}{a_{k}} \prod_{i=v}^{n} x_{i} \leqq \frac{1}{a_{k}} \prod_{i=v}^{n} a_{i} \\
& =\prod_{i=v}^{k-1} a_{i} \cdot \prod_{i=k+1}^{n} a_{i}=\prod_{i=v}^{n-1} b_{i} .
\end{aligned}
$$

Therefore we can apply the assumption of induction and deduce that

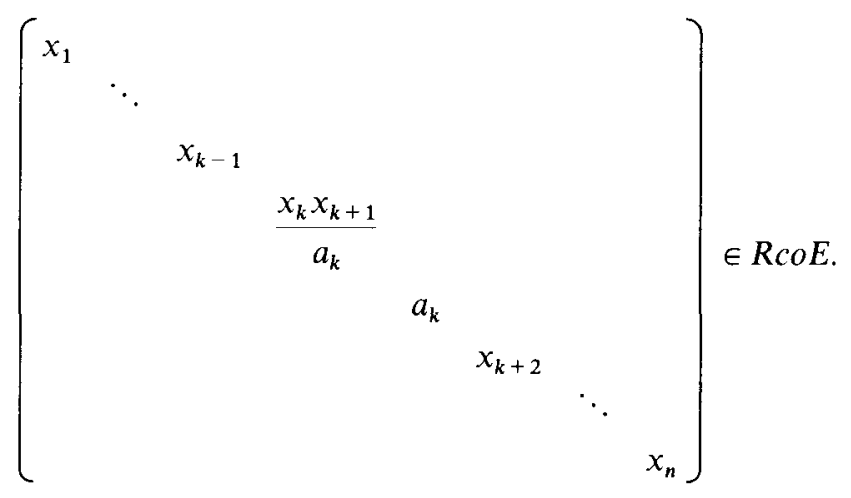

As above, we get that

$$
A_{ \pm} \in R \operatorname{co} E
$$

and, finally, combining (3.19) and (3.21), we obtain the claimed result:

$\xi \in R \operatorname{RoE}$.

Part 3.2 $\left\{\begin{array}{l}a_{k} \leqq x_{k} \leqq x_{k+1} \leqq a_{k+1}, \\ \prod_{i=\mu}^{k-1} x_{i} \cdot \prod_{i=k+2}^{n} x_{i} \leqq \prod_{i=\mu+1}^{k} a_{i} \cdot \prod_{i=k+2}^{n} a_{i} \quad \mu=1, \ldots, k-1 .\end{array}\right.$

We choose here $b=a_{k+1}$ in (3.19) and (3.20); therefore we can find $O_{ \pm}, O_{ \pm}^{\prime} \in O(n)$ such that

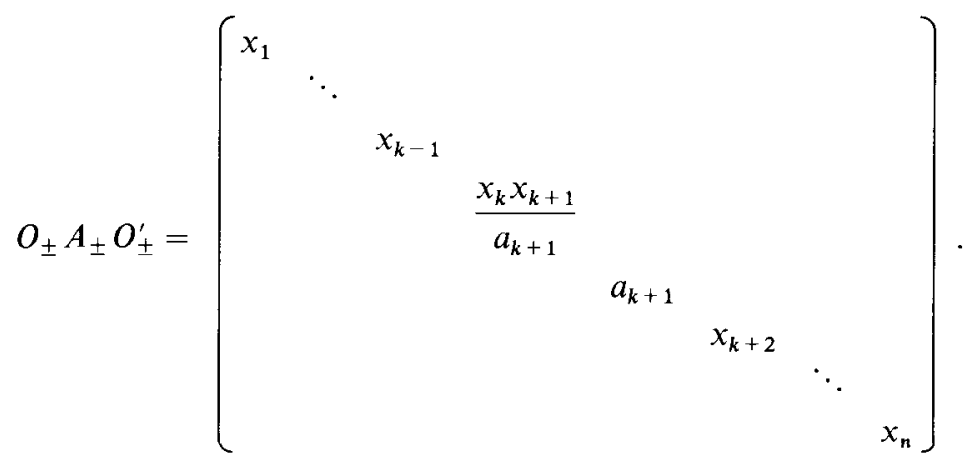

We have to prove the hypothesis of induction for

$$
\left\{y_{1}=x_{1}, \ldots, y_{k-1}=x_{k-1}, y_{k}=\frac{x_{k} x_{k+1}}{a_{k+1}}, y_{k+1}=x_{k+2}, \ldots, y_{n-1}=x_{n}\right\}
$$


and for

$$
\left\{b_{1}=a_{1}, \ldots, b_{k}=a_{k}, b_{k+1}=a_{k+2}, \ldots, b_{n-1}=a_{n}\right\} .
$$

Observe that, since $x_{k+1} \leqq a_{k+1}$, then $y_{k} \leqq y_{k+1} \leqq \ldots \leqq y_{n-1}$. On the contrary, a priori, we cannot compare $y_{k}$ to $y_{1} \leqq \ldots \leqq y_{k-1}$. We verify the hypothesis of induction. From the assumption $\xi \in X$ and from that of Part 3.2 we can write:

(1) if $v \geqq k+1$, then $\Pi_{i=v}^{n-1} y_{i}=\prod_{i=v+1}^{n} x_{i} \leqq \Pi_{i=v+1}^{n} a_{i}=\prod_{i=v}^{n-1} b_{i}$;

(2) if $v=k$, then

$$
\left\{\begin{array}{l}
\prod_{i=k}^{n-1} y_{i}=\frac{1}{a_{k+1}} \prod_{i=k}^{n} x_{i} \leqq \frac{1}{a_{k+1}} \prod_{i=k}^{n} a_{i}=a_{k} \prod_{i=k+2}^{n} a_{i}=b_{k} \prod_{i=k+1}^{n-1} b_{i}=\prod_{i=k}^{n-1} b_{i} \\
y_{k-1} \prod_{i=k+1}^{n-1} y_{i}=x_{k-1} \prod_{i=k+2}^{n} x_{i} \leqq a_{k} \prod_{i=k+2}^{n} a_{i}=\prod_{i=k}^{n-1} b_{i}
\end{array}\right.
$$

(3) if $k-1 \geqq v \geqq 1$, then

$$
\left\{\begin{aligned}
\prod_{i=v}^{n-1} y_{i} & =\prod_{i=v}^{k-1} x_{i} \frac{x_{k} x_{k+1}}{a_{k+1}} \cdot \prod_{i=k+2}^{n} x_{i}=\frac{1}{a_{k+1}} \cdot \prod_{i=v}^{n} x_{i} \\
& \leqq \frac{1}{a_{k+1}} \cdot \prod_{i=v}^{n} a_{i}=\prod_{i=v}^{k} a_{i} \cdot \prod_{i=k+2}^{n} a_{i}=\prod_{i=v}^{n-1} b_{i}, \\
\prod_{i=v-1}^{k-1} y_{i} \cdot \prod_{i=k+1}^{n-1} y_{i} & =\prod_{i=v-1}^{k-1} x_{i} \cdot \prod_{i=k+2}^{n} x_{i} \\
& \leqq \prod_{i=v}^{k} a_{i} \cdot \prod_{i=k+2}^{n} a_{i}=\prod_{i=v}^{k} b_{i} \cdot \prod_{i=k+1}^{n-1} b_{i}=\prod_{i=v}^{n-1} b_{i} .
\end{aligned}\right.
$$

We can apply the hypothesis of induction and deduce that

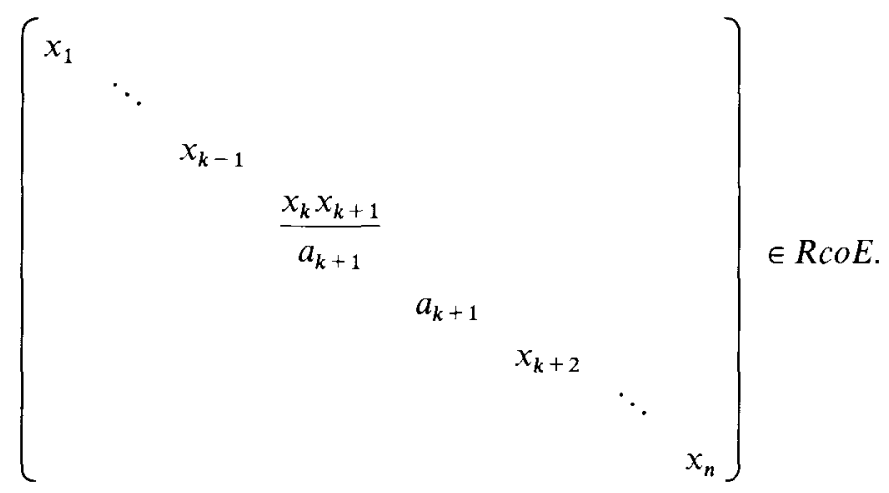

Since $R c o E$ is invariant up the orthogonal transformations, we can obtain that

$$
A_{ \pm} \in R \operatorname{Ro} E
$$

Finally, combining (3.19) and (3.22), we can write $\xi \in R c o E$. In conclusion, we have obtained the claimed result: $X \subset R c o E$. 
Proof of Theorem 3.1(iii). Let $Y=\left\{\xi \in \mathbf{R}^{n \times n}: \prod_{i=v}^{n} \lambda_{i}(\xi)<\Pi_{i=v}^{n} a_{i}, v=1, \ldots, n\right\}$. We show that int $R \operatorname{co} E=Y$. We divide the proof into two steps.

Step 1. $Y \subset$ int $R c o E$, since by continuity $Y$ is open and, by (ii), $Y \subset R c o E$.

Step 2. int $R c o E \subset Y$. So let $\xi \in$ int $R c o E$; we can therefore find $\varepsilon$ sufficiently small so that $B_{\varepsilon}(\xi) \subset R \operatorname{co} E$ (where $B_{\varepsilon}(\xi)$ denotes the ball centred at $\xi$ and of radius $\varepsilon$ ). Let $R, R^{\prime}$ be orthogonal matrices so that

$$
\xi=R\left(\begin{array}{llll}
\lambda_{1}(\xi) & & & \\
& \lambda_{2}(\xi) & & \\
& & \ddots & \\
& & & \lambda_{n}(\xi)
\end{array}\right) R^{\prime} .
$$

Define

$$
\eta=R\left(\begin{array}{llll}
\lambda_{1}(\xi) & & & \\
& \lambda_{2}(\xi) & & \\
& & \ddots & \\
& & & \lambda_{n}(\xi)+\frac{\varepsilon}{2}
\end{array}\right) R^{\prime} .
$$

Since $|\eta-\xi|=(\varepsilon / 2)<\varepsilon$, then $\eta \in R \operatorname{coE}$. We then get

$$
\lambda_{n}(\xi)<\lambda_{n}(\eta) \leqq a_{n} .
$$

Assume that $\lambda_{v}(\xi) \neq 0$ for every $v$; we then get for $v=1, \ldots, n$ and with the convention $\prod_{i=n+1}^{n} \lambda_{i}(\xi)=1$,

$$
\prod_{i=v}^{n} \lambda_{i}(\xi)=\prod_{i=v}^{n-1} \lambda_{i}(\xi) \cdot \lambda_{n}(\xi)<\prod_{i=v}^{n-1} \lambda_{i}(\eta) \cdot \lambda_{n}(\eta) \leqq \prod_{i=v}^{n-1} a_{i} \cdot a_{n}
$$

which implies that $\xi \in Y$.

Finally, if $\exists \bar{v} \in\{1, \ldots, n\}$ such that $\lambda_{\bar{v}}(\xi)=0$, and $\lambda_{\bar{v}+1}(\xi)>0$, then the same argument as above is valid for $v=\bar{v}+1, \ldots, n$ and is trivial if $v=1, \ldots, \bar{v}$. We therefore also get that $\xi \in Y$.

REMARK 3.3. We should draw the attention to the following facts.

(1) We have privileged proofs that are as similar as possible for $c o E$ and $R c o E$, replacing $\Sigma$ by $\Pi$. We did not succeed in doing this for the case $n=2$.

(2) The above choice forced us, in the convex case, to consider nondiagonal (but symmetric) decompositions of the matrix $\xi$. If one insists in keeping decompositions with only diagonal matrices, then this is possible and is indeed achieved here for $n=2$.

\section{Acknowledgments}

We would like to thank P. Marcellini for important discussions on this paper. Part of this research was financially supported by Fonds National Suisse (21-50472.97). 


\section{References}

1 G. Aubert and R. Tahraoui. Sur la faible fermeture de certains ensembles de contraintes en élasticité non linéaire plan. C. R. Acad. Sci. Paris 290 (1980), 537-40.

2 J. M. Ball. Convexity conditions and existence theorems in nonlinear elasticity. Arch. Rational Mech. Anal. 63 (1977), 337-403.

3 B. Dacorogna. Direct Methods in the Calculus of Variations. Applied Mathematical Sciences 78 (New York: Springer, 1989).

4 B. Dacorogna and P. Marcellini. Théorème d'existence dans le cas scalaire et vectoriel pour les équations de Hamilton-Jacobi. C. R. Acad. Sci. Paris. Sér. I 322 (1996), 237-40.

5 B. Dacorogna and P. Marcellini. Sur le problème de Cauchy-Dirichlet pour les systèmes d'équations non linéaires du premier ordre. C. R. Acad. Sci. Paris Sér. I 323 (1996), 599-602.

6 B. Dacorogna and P. Marcellini. General existence theorems for Hamilton-Jacobi equations in the scalar and vectorial cases. Acta Math. 178 (1997), 1-37.

7 B. Dacorogna and P. Marcellini. Cauchy-Dirichlet problem for first order nonlinear systems. J. Funct. Anal. 152 (1998), 404-46.

8 H. Le Dret. Sur les fonctions de matrices convexes et isotropes. C. R. Acad. Sci. Paris Sér. I 310 (1990), 617-20.

9 R. T. Rockafellar. Convex analysis (Princeton, NJ: Princeton University Press, 1970).

(Issued 18 December 1998) 